\title{
ON THE SINGULARITIES OF THE CONTINUOUS JACOBI TRANSFORM WHEN $\alpha+\beta=0$
}

\section{AHMED I. ZAYED}

ABSTRACT. Let $\alpha, \beta>-1$ and $P_{\lambda}^{(\alpha, \beta)}(x)=(1-x)^{\alpha}(1+x)^{\beta} P_{\lambda}^{(\alpha, \beta)}(x)$, where $P_{\lambda}^{(\alpha, \beta)}(x)$ is the Jacobi function of the first kind, $\lambda \geq-(\alpha+\beta+1) / 2$, and $-1<x \leq 1$. Let

$$
F^{(\alpha, \beta)}(\lambda)=\frac{1}{2^{\alpha+\beta+1}}\left\langle f(x), \mathcal{P}_{\lambda}^{(\alpha, \beta)}(x)\right\rangle=\frac{1}{2^{\alpha+\beta+1}} \int_{-1}^{1} f(x) \mathcal{P}_{\lambda}^{(\alpha, \beta)}(x) d x
$$

whenever the integral exists. It is known that for $\alpha+\beta=0$, we have

$$
f(x)=\lim _{n \rightarrow \infty} 4 \int_{0}^{n} F^{(\alpha, \beta)}\left(\lambda-\frac{1}{2}\right) P_{\lambda-1 / 2}^{(\beta, \alpha)}(-x) \lambda
$$

$$
\times \sin \pi \lambda \frac{\Gamma^{2}(\lambda+1 / 2)}{\Gamma(\lambda+\alpha+1 / 2) \Gamma(\lambda+\beta+1 / 2)} d \lambda
$$

almost everywhere in $[-1,1]$.

In this paper, we devise a technique to continue $f(x)$ analytically to the complex $z$-plane and locate the singularities of $f(z)$ by relating them to the singularities of

$$
g(t)=\int_{0}^{\infty} e^{-\lambda t} F^{(\alpha, \beta)}(\lambda) \frac{d \lambda}{\Gamma(\lambda+\alpha+1)} .
$$

However, this will be done in the more general case where the limit in (*) exists in the sense of Schwartz distributions and defines a generalized function $f(x)$. In this case, we pass from $f(x)$ to its analytic representation

$$
\hat{f}(z)=\frac{1}{2 \pi i}\left\langle f(x), \frac{1}{x-z}\right\rangle, \quad z \notin \operatorname{supp} f,
$$

and then relate the singularities of $\hat{f}(z)$ to those of $g(t)$.

1. Introduction. In an earlier paper [5] Z. Nehari devised a technique to locate the singular points of the function

$$
f(t)=\sum_{n=0}^{\infty} \hat{f}(n) P_{n}(t), \quad|t+1|+|t-1|<\frac{1}{\alpha}+\alpha
$$

by relating them to the singular points of the associated power series

$$
g(z)=\sum_{n=0}^{\infty} \hat{f}(n) z^{n}, \quad|z|<\alpha
$$

where

$$
\hat{f}(n)=\left(n+\frac{1}{2}\right) \int_{-1}^{1} f(t) P_{n}(t) d t,
$$

Received by the editors May 21, 1986.

1980 Mathematics Subject Classification (1985 Revision). Primary 44A15; Secondary 30B40.

Key words and phrases. Continuous Jacobi transform, analytic continuation, singular points. 
$\varlimsup_{n \rightarrow \infty}|\hat{f}(n)|^{1 / n}=1 / \alpha<1$, and $P_{n}(t)$ is the Legendre polynomial of degree $n$.

It was shown that $f(t)$ has a singular point at $t=t_{0}$ if and only if $g(z)$ has one at $z=z_{0}$ where $t_{0}=\frac{1}{2}\left(z_{0}+1 / z_{0}\right), t_{0} \neq \pm 1$.

This result has been generalized in a number of different ways. For example, in [3], R. Gilbert replaced the Legendre polynomials in (1.1) by the Jacobi polynomials $P_{n}^{(\alpha, \beta)}(t)\left(P_{n}^{(0,0)}(t)=P_{n}(t)\right)$, while in [7], G. Walter relaxed the restriction on $\{\hat{f}(n)\}_{n=0}^{\infty}$ so that $\varlimsup_{n \rightarrow \infty}|\hat{f}(n)|^{1 / n}=1$. In the latter case, the series (1.1) does not converge in the classical sense, nevertheless, it was shown that it converges in the sense of Schwartz distributions to a generalized function $f(t)$ and that the singularities of the analytic representation $\hat{f}(t)$ of $f(t)$ can be related to the singularities of $g(z)$ as in Nehari's result.

Another generalization was developed in [8], where the discrete Legendre transform (1.3) was replaced by the continuous Legendre transform

$$
\hat{f}(\lambda)=\frac{1}{2} \int_{-1}^{1} f(t) P_{\lambda}(t) d t, \quad \lambda \in\left[-\frac{1}{2}, \infty\right),
$$

where $P_{\lambda}(t)$ is the Legendre function with continuous parameter $\lambda$ which reduces to $P_{n}(t)$ if $\lambda$ is a nonnegative integer $n$. It was shown that the analogue of (1.1), i.e.,

$$
f(t)=4 \int_{0}^{\infty} \hat{f}\left(\lambda-\frac{1}{2}\right) P_{\lambda-1 / 2}(-t) \lambda \sin \pi \lambda d \lambda,
$$

has a singular point at $t=t_{0}$ if and only if the analogue of (1.2), i.e.,

$$
g(z)=\int_{0}^{\infty} e^{-z \lambda} \hat{f}\left(\lambda-\frac{1}{2}\right) d \lambda
$$

has one at $z=z_{0}$ where $t_{0}=\frac{1}{2}\left(e^{z_{0}}+e^{-z_{0}}\right)$.

The purpose of this paper is not only to extend the results of [8] to the continuous Jacobi transform but also to the case where the integral in (1.5) converges in the sense of Schwartz distributions to a generalized function $f(t)$. More precisely, we shall replace the Legendre function in (1.5) by the Jacobi function and relax the convergence of the integral in (1.5) so that $f(t)$ will merely exist as a generalized function. We then relate the singularities of its analytic representation $\hat{f}(t)$ to those of $g(z)$ given in (1.6).

2. Preliminaries. In this section, we introduce some of the formulas and notation that will be used in the sequel and refer the reader to $[\mathbf{2}, \mathbf{6}, \mathbf{9}, \mathbf{1 0}]$ for more details.

Let $\alpha, \beta>-1, \lambda \in \mathbf{R}$, and $\lambda+\alpha+1 \neq 0,-1,-2, \ldots$ Then the Jacobi function $P_{\lambda}^{(\alpha, \beta)}(x)$ of the first kind is given by

$$
\begin{aligned}
& P_{\lambda}^{(\alpha, \beta)}(x)=\frac{\Gamma(\lambda+\alpha+1)}{\Gamma(\alpha+1) \Gamma(\lambda+1)}{ }_{2} F_{1}\left(-\lambda, \lambda+\alpha+\beta+1 ; \alpha+1 ; \frac{1-x}{2}\right) \\
& x \in(-1,1]
\end{aligned}
$$

where ${ }_{2} F_{1}(a, b ; c ; z)$ is the hypergeometric function. When $\lambda$ is a nonnegative integer $n, P_{n}^{(\alpha, \beta)}(x)$ becomes the Jacobi polynomial of degree $n$. 
Let

$$
P_{\lambda}^{(\alpha, \beta)}(x)=(1-x)^{\alpha}(1+x)^{\beta} P_{\lambda}^{(\alpha, \beta)}(x)
$$

and

$$
\mathcal{L}_{x}=\left(1-x^{2}\right) \frac{d^{2}}{d x^{2}}+[(\alpha-\beta)+(\alpha+\beta-2) x] \frac{d}{d x}+(\alpha+\beta) .
$$

Then it is easy to show that

$$
\mathcal{L}_{x} P_{\lambda}^{(\alpha, \beta)}(x)=\eta(\lambda) P_{\lambda}^{(\alpha, \beta)}(x),
$$

where

$$
\eta(\lambda)=-\lambda(\lambda+\alpha+\beta+1) .
$$

Moreover, we have the following recurrence relation:

$$
\begin{aligned}
2 \lambda(\lambda+ & \alpha+\beta)(2 \lambda+\alpha+\beta-2) P_{\lambda}^{(\alpha, \beta)}(x) \\
= & (2 \lambda+\alpha+\beta-1)\left\{(2 \lambda+\alpha+\beta)(2 \lambda+\alpha+\beta-2) x+\alpha^{2}-\beta^{2}\right\} \\
& \times P_{\lambda-1}^{(\alpha, \beta)}(x)-2(\lambda+\alpha-1)(\lambda+\beta-1)(2 \lambda+\alpha+\beta) P_{\lambda-2}^{(\alpha, \beta)}(x) .
\end{aligned}
$$

The Jacobi function of the second kind is defined by

$$
q_{\lambda}^{(\alpha, \beta)}(z)=\frac{1}{2}(z-1)^{-\alpha}(z+1)^{-\beta} \int_{-1}^{1} \frac{P_{\lambda}^{(\alpha, \beta)}(t)}{z-t} d t
$$

where $z$ is in the complex plane cut along $[-1,1]$.

From Theorem 9.2.1 in [6], we obtain that

$$
\frac{(1-t)^{\alpha}(1+t)^{\beta}}{2(z-t)}=(z-1)^{\alpha}(z+1)^{\beta} \sum_{n=0}^{\infty} \frac{1}{h_{n}} \rho_{n}^{(\alpha, \beta)}(t) q_{n}^{(\alpha, \beta)}(z)
$$

where

$$
h_{n}=\frac{\Gamma(n+\alpha+1) \Gamma(n+\beta+1)}{n ! \Gamma(2 n+\alpha+\beta+1) \Gamma(n+\alpha+\beta+1)},
$$

$t$ lies in the interior of, and $z$ in the exterior of, an arbitrary ellipse with foci at \pm 1 .

The following estimates will be needed: Let $c>0$ and

$$
\kappa(\lambda)= \begin{cases}\sup _{x \in[-1,1]}\left|(1+x)^{c} P_{\lambda}^{(\alpha, \beta)}(x)\right| & \text { if }-1<\beta \leq 0, \\ \sup _{x \in[-1,1]}\left|(1+x)^{\beta+c} P_{\lambda}^{(\alpha, \beta)}(x)\right| & \text { if } \beta \geq 0 .\end{cases}
$$

Then $\kappa(\lambda)=O\left(\lambda^{q}\right)$ as $\lambda \rightarrow \infty$ where $q=\max (\alpha, \beta)$. Moreover, for $z$ in the complex plane cut along $[-1,1]$, we have

$$
q_{\lambda}^{(\alpha, \beta)}(z)=O\left(|\zeta|^{\lambda}\right) \quad \text { as } \lambda \rightarrow \infty,
$$

where $\zeta=z-\sqrt{z^{2}-1}$ and $|\zeta|<1$.

Let $I$ denote the open interval $(-1,1)$. For fixed $\alpha, \beta>-1$, we define the space $\mathscr{H}_{\alpha, \beta}$ as the space of all infinitely differentiable functions $\phi(x)$ on $I$ such that

$$
\gamma_{k, c}(\phi)=\sup _{x \in I}\left|(1-x)^{-\alpha}(1+x)^{c} \mathcal{L}_{x}^{k} \phi(x)\right|<\infty
$$

for any nonnegative integer $k$ and any $c$ with $\max (-\beta, 0)<c<1$. 
In [9], we showed that when provided with the topology generated by the seminorms $\left\{\gamma_{k, c}\right\}, \mathfrak{H}_{\alpha, \beta}$ becomes a Fréchet space whose dual $\mathfrak{H}_{\alpha, \beta}^{*}$ is a subspace of $D^{*}(I)$, the space of Schwartz distributions defined on $I$. Since $P_{\lambda}^{(\alpha, \beta)}(x) \in \mathcal{H}_{\alpha, \beta}$, we can define the continuous Jacobi transform $F^{(\alpha, \beta)}(\lambda)$ of $f(x) \in \mathcal{H}_{\alpha, \beta}^{*}$ by

$$
F^{(\alpha, \beta)}(\lambda)=\frac{1}{2^{\alpha+\beta+1}}\left\langle f(x), \mathcal{P}_{\lambda}^{(\alpha, \beta)}(x)\right\rangle .
$$

Furthermore, in the sense of convergence in $D^{*}(I)$, we have the inversion formula [9, Theorem 5.1]

$$
f(x)=\lim _{n \rightarrow \infty} 4 \int_{0}^{n} F^{(\alpha, \beta)}\left(\lambda-\frac{1}{2}\right) P_{\lambda-1 / 2}^{(\beta, \alpha)}(-x) H_{0}(\lambda) \lambda \sin \pi \lambda d \lambda,
$$

where

$$
H_{0}(\lambda)=\frac{\Gamma^{2}(\lambda+1 / 2)}{\Gamma(\lambda+\alpha+1 / 2) \Gamma(\lambda+\beta+1 / 2)}
$$

provided that $\alpha+\beta=0$. It was also shown that [9, Theorem 4.1]

$$
F^{(\alpha, \beta)}(\lambda)=O\left(\lambda^{m}\right) \quad \text { as } \lambda \rightarrow \infty \text { for some integer } m \text {. }
$$

Finally, if $f(x)$ is a generalized function with compact support, then the analytic representation $\hat{f}(z)$ of $f(x)$ is defined by

$$
\hat{f}(z)=\frac{1}{2 \pi i}\left\langle f(x), \frac{1}{x-z}\right\rangle, \quad z \notin \operatorname{supp} f .
$$

Moreover, if $\phi(x) \in C^{\infty}(I)$, then

$$
\lim _{\varepsilon \rightarrow 0^{+}} \int_{-\infty}^{\infty}[\hat{f}(x+i \varepsilon)-\hat{f}(x-i \varepsilon)] \phi(x) d x=\langle f(x), \phi(x)\rangle
$$

(see [1]).

\section{Analytic representation for elements of $\mathfrak{K}_{\alpha, \beta}^{*}$.}

Proposition 3.1. Let $f(t) \in \mathcal{H}_{\alpha, \beta}^{*}$ and set

$$
f^{(\alpha, \beta)}(t)=(1-t)^{\alpha}(1+t)^{\beta} f(t) .
$$

Then,

(i) the analytic representation $\hat{f}^{(\alpha, \beta)}(z)$ of $f^{(\alpha, \beta)}(t)$ is well defined;

(ii) in addition to that, if the support of $f(t)$ is contained in $(-1,1)$, then $\hat{f}^{(\alpha, \beta)}(z)$ has the representation

$$
\hat{f}^{(\alpha, \beta)}(z)=\frac{2}{\pi i} \int_{0}^{\infty} F^{(\alpha, \beta)}\left(\lambda-\frac{1}{2}\right) Q_{\lambda-1 / 2}^{(\beta, \alpha)}(z) H_{0}(\lambda) \lambda \sin \pi \lambda d \lambda, \quad z \notin \operatorname{supp} f,
$$

where

$$
Q_{\lambda-1 / 2}^{(\beta, \alpha)}(z)=2(-1)^{\beta}(1-z)^{\alpha}(1+z)^{\beta} q_{\lambda-1 / 2}^{(\beta, \alpha)}(-z)
$$


$q_{\lambda-1 / 2}^{(\beta, \alpha)}(-z), F^{(\alpha, \beta)}\left(\lambda-\frac{1}{2}\right)$ are given by (2.7) and (2.13). Moreover, the integral in (3.2) converges absolutely and uniformly on any compact subset of the complex plane cut along $[-1,1]$.

PROOF. (i) From (2.17), we have for $z \notin \operatorname{supp} f$,

$$
\hat{f}^{(\alpha, \beta)}(z)=\frac{1}{2 \pi i}\left\langle f^{(\alpha, \beta)}(t), \frac{1}{t-z}\right\rangle=\frac{1}{2 \pi i}\left\langle f(t), \frac{(1-t)^{\alpha}(1+t)^{\beta}}{t-z}\right\rangle .
$$

For (3.4) to make sense, we must show that

$$
\frac{(1-t)^{\alpha}(1+t)^{\beta}}{(t-z)} \in \not_{\alpha, \beta} .
$$

Upon applying the operator $\mathcal{L}_{t}^{k}$ to both sides of (2.8) and using (2.4), we obtain

$$
\mathcal{L}_{t}^{k}\left(\frac{(1-t)^{\alpha}(1+t)^{\beta}}{(z-t)}\right)=\sum_{n=0}^{\infty} \frac{[\eta(n)]^{k}}{h_{n}} \mathcal{P}_{n}^{(\alpha, \beta)}(t) Q_{n}^{(\alpha, \beta)}(-z)<\infty
$$

for any nonnegative integer $k$. The series in (3.6) converges in view of (2.5), (2.9), (2.10), and (2.11).

From (2.10), (2.12), and (3.6), we deduce that

$$
\gamma_{k, c}\left(\frac{(1-t)^{\alpha}(1+t)^{\beta}}{z-t}\right)<\infty
$$

which proves (3.5).

(ii) Let

$$
f_{n}^{(\alpha, \beta)}(t)=(1-t)^{\alpha}(1+t)^{\beta} f_{n}(t), \quad n=1,2,3, \ldots
$$

where

$$
f_{n}(t)=4 \int_{0}^{n} F^{(\alpha, \beta)}\left(\lambda-\frac{1}{2}\right) P_{\lambda-1 / 2}^{(\beta, \alpha)}(-t) H_{0}(\lambda) \lambda \sin \pi \lambda d \lambda .
$$

Appealing to (3.4), (3.8), and (3.9), we can write the analytic representation $\hat{f}_{n}^{(\alpha, \beta)}(z)$ of $f_{n}^{(\alpha, \beta)}(t)$ in the form

$$
\begin{aligned}
& \hat{f}_{n}^{(\alpha, \beta)}(z)= \frac{1}{2 \pi i}\left\langle f_{n}^{(\alpha, \beta)}(t), \frac{1}{t-z}\right\rangle \\
&=\frac{2}{\pi i} \int_{-1}^{1} \frac{1}{t-z}\left\{\int_{0}^{n} F^{(\alpha, \beta)}\left(\lambda-\frac{1}{2}\right)(1-t)^{\alpha}(1+t)^{\beta}\right. \\
&\left.\quad \quad \times P_{\lambda-1 / 2}^{(\beta, \alpha)}(-t) H_{0}(\lambda) \lambda \sin \pi \lambda d \lambda\right\} d t \\
&=\frac{2}{\pi i} \int_{0}^{n} F^{(\alpha, \beta)}\left(\lambda-\frac{1}{2}\right) Q_{\lambda-1 / 2}^{(\beta, \alpha)}(z) H_{0}(\lambda) \lambda \sin \pi \lambda d \lambda,
\end{aligned}
$$

where $Q_{\lambda-1 / 2}^{(\beta, \alpha)}(z)$ is given by (3.3).

From (2.14), it follows that since $f(t)$ has support in $(-1,1)$, then so does $f_{n}(t)$ for all $n$. Moreover, $\operatorname{supp} f_{n} \subseteq \operatorname{supp} f$ for all $n$. 
Let $\chi(t) \in D(I)$ such that $\chi(t)=1$ on some neighborhood of the support of $f(t)$. Then, by Corollary 5.1 in [9] and (2.14), we have

$$
\begin{aligned}
\lim _{n \rightarrow \infty} \hat{f}_{n}^{(\alpha, \beta)}(z) & =\frac{1}{2 \pi i} \lim _{n \rightarrow \infty}\left\langle\hat{f}_{n}^{(\alpha, \beta)}(t), \frac{1}{t-z}\right\rangle=\frac{1}{2 \pi i} \lim _{n \rightarrow \infty}\left\langle\hat{f}_{n}^{(\alpha, \beta)}(t), \frac{\chi(t)}{t-z}\right\rangle \\
& =\frac{1}{2 \pi i}\left\langle\hat{f}^{(\alpha, \beta)}(t), \frac{\chi(t)}{t-z}\right\rangle=\frac{1}{2 \pi i}\left\langle\hat{f}^{(\alpha, \beta)}(t), \frac{1}{t-z}\right\rangle=\hat{f}^{(\alpha, \beta)}(z),
\end{aligned}
$$

which, together with (3.10), implies (3.2).

The convergence of the integral in (3.2) as described above can be seen from (2.16) and (2.11). Q.E.D.

4. The singularity theorem. Throughout the rest of this paper, we shall assume that $\alpha+\beta=0$. To prove the main result of this paper which is Theorem 4.1 , we need the following lemma.

LEMMA 4.1. Let

$$
J(t, z)=\int_{0}^{\infty} e^{-\lambda t} P_{\lambda}^{(\alpha, \beta)}(z) d \Lambda
$$

and

$$
I(t, z)=\int_{0}^{\infty} e^{-\lambda t} Q_{\lambda}^{(\beta, \alpha)}(z) \cos \pi \lambda d \sigma(\lambda),
$$

where $\operatorname{Re} t>0, z$ is in the complex plane cut along $[-1,1], d \Lambda=d \lambda / \Gamma(\lambda+\alpha+1)$, and $d \sigma(\lambda)=H_{0}\left(\lambda+\frac{1}{2}\right)\left(\lambda+\frac{1}{2}\right) \Gamma(\lambda+\alpha+1) d \lambda$. Then both $J(t, z)$ and $I(t, z)$ can be continued analytically so that their only possible singularities are at $z \pm 1$ and $z=\frac{1}{2}\left(e^{t}+e^{-t}\right)$.

Proof. First, we prove the lemma for $J(t, z)$. From (4.1), we obtain that

$$
\frac{d}{d t}\left(e^{t} \frac{d J}{d t}\right)=e^{t} \int_{0}^{\infty} \lambda(\lambda-1) e^{-\lambda t} p_{\lambda}^{(\alpha, \beta)}(z) d \Lambda,
$$

which upon using (2.6) (for $\alpha+\beta=0$ ) yields

$$
\begin{aligned}
e^{-t} \frac{d}{d t}\left(e^{t} \frac{d J}{d t}\right)= & \int_{0}^{\infty}(2 \lambda-1)(\lambda-1) z e^{-\lambda t} P_{\lambda-1}^{(\alpha, \beta)}(z) d \Lambda \\
& -\int_{0}^{\infty}(\lambda-1)^{2} e^{-\lambda t} P_{\lambda-2}^{(\alpha, \beta)}(z) d \Lambda+\alpha^{2} \int_{0}^{\infty} e^{-\lambda t} P_{\lambda-2}^{(\alpha, \beta)}(z) d \Lambda \\
= & \mathrm{I}_{1}-\mathrm{I}_{2}+\mathrm{I}_{3},
\end{aligned}
$$

where $I_{1}, I_{2}$, and $I_{3}$ denote the three integrals on the right-hand side respectively. By changing the variables in these three integrals, we obtain

$$
\begin{aligned}
\mathrm{I}_{1} & =z \int_{-1}^{0}(2 \lambda+1) \lambda e^{-(\lambda+1) t} p_{\lambda}^{(\alpha, \beta)}(z) d \Lambda+z \int_{0}^{\infty}(2 \lambda+1) \lambda e^{-(\lambda+1) t} p_{\lambda}^{(\alpha, \beta)}(z) d \Lambda \\
& =h_{1}(t, z)+z e^{-t}\left(2 \frac{d^{2} J}{d t^{2}}-\frac{d J}{d t}\right)
\end{aligned}
$$




$$
\begin{aligned}
\mathrm{I}_{2} & =\int_{-2}^{0}(\lambda+1)^{2} e^{-(\lambda+2) t} P_{\lambda}^{(\alpha, \beta)}(z) d \lambda+e^{-t} \frac{d^{2}}{d t^{2}}\left(e^{-t} J\right) \\
& =h_{2}(t, z)+e^{-t} \frac{d^{2}}{d t^{2}}\left(e^{-t} J\right),
\end{aligned}
$$

and

$$
\begin{aligned}
\mathrm{I}_{3} & =\alpha^{2}\left[\int_{-2}^{0} e^{-(\lambda+2) t} p_{\lambda}^{(\alpha, \beta)}(z) d \Lambda+\int_{0}^{\infty} e^{-(\lambda+2) t} p_{\lambda}^{(\alpha, \beta)}(z) d \Lambda\right] \\
& =h_{3}(t, z)+\alpha^{2} e^{-2 t} J
\end{aligned}
$$

where $h_{1}, h_{2}$, and $h_{3}$ are analytic functions in $t$ and $z$ except for possible singularities at $z= \pm 1$. By combining (4.3)-(4.6), we obtain that

$$
e^{-t} \frac{d}{d t}\left(e^{t} \frac{d J}{d t}\right)=z e^{-t}\left[2 \frac{d^{2} J}{d t^{2}}-\frac{d J}{d t}\right]-e^{-t} \frac{d^{2}}{d t^{2}}\left(e^{-t} J\right)+\alpha^{2} e^{-2 t} J+h(t, z)
$$

which, after some simplifications, yields

$$
\left(e^{t}-2 z+e^{-t}\right) \frac{d^{2} J}{d t^{2}}+\left(e^{t}+z-2 e^{-t}\right) \frac{d J}{d t}+\left(1-\alpha^{2}\right) e^{-t} J=e^{t} h(t, z)
$$

where $h(t, z)$ is an analytic function in $t$ and $z$ except for possible singularities at $z= \pm 1$. The conclusion of the lemma now follows from the fact that the solution of the differential equation (4.7) is analytic everywhere, except possibly at $z= \pm 1$ and $e^{t}-2 z+e^{-t}=0$, i.e., $z=\frac{1}{2}\left(e^{t}+e^{-t}\right)$.

The proof for $I(t, z)$ is similar since $Q_{\lambda}^{(\beta, \alpha)}(z)$ satisfies the same recurrence relation $(2.6)$ as $P_{\lambda}^{(\alpha, \beta)}(z)$. Q.E.D.

COROLLARY 4.1. Let

$$
L(-t, z)=\frac{2}{\pi i} \int_{0}^{\infty} e^{-(\lambda-1 / 2) t} Q_{\lambda-1 / 2}^{(\beta, \alpha)}(z) H_{0}(\lambda) \Gamma\left(\lambda+\alpha+\frac{1}{2}\right) \lambda \sin \pi \lambda d \lambda,
$$

where $\operatorname{Re} t>0$ and $z$ is in the complex plane cut along $[-1,1]$. Then, $L(t, z)$ can be continued analytically so that its only possible singularities are at $z= \pm 1$ and $z=\frac{1}{2}\left(e^{t}+e^{-t}\right)$.

PROOF. Upon changing the variable of integration in (4.8) from $\lambda$ to $\lambda+\frac{1}{2}$, we obtain that

$$
L(-t, z)=\frac{2}{\pi i} I(t, z)+\Phi_{1}(t, z)
$$

where $\Phi_{1}(t, z)$ is a function' that is analytic everywhere except possibly at $z= \pm 1$. The proof now follows immediately from Lemma 4.1.

We remark that the integral representation (4.8) of $L(-t, z)$ also exists for $\operatorname{Re} t \leq$ 0 provided that $\operatorname{Re} t>\ln |\varsigma|$ where $z$ and $\varsigma$ are related as in (2.11).

THEOREM 4.1. Let $f(x) \in \mathcal{H}_{\alpha, \beta}^{*}$ have support in $(-1,1)$. Let $F^{(\alpha, \beta)}(\lambda)$ be the continuous Jacobi transform of $f(x)$ and let $f^{(\alpha, \beta)}(x)$ be given as in (3.1). Then the analytic representation $\hat{f}^{(\alpha, \beta)}(z)$ of $f^{(\alpha, \beta)}(x)$ has a singularity at the point $z=z_{0} \in(-1,1)$ if and only if the analytic function

$$
g(t)=\int_{0}^{\infty} e^{-t \lambda} F^{(\alpha, \beta)}(\lambda) d \Lambda
$$


has a singularity at either the point $t=t_{0}$ or $t=\bar{t}_{0}$ where $z_{0}=\frac{1}{2}\left(e^{t_{0}}+e^{-t_{0}}\right)$ and $t_{0} \in(-i \pi, i \pi)$.

PROOF. The main idea of the proof is to construct two integral operators; one to map $\hat{f}(z)$ into $g(t)$ and the other to map $g(t)$ into $\hat{f}^{(\alpha, \beta)}(z)$, then apply Hadamard's argument. First, let us observe that $g(t)$ is analytic in Ret $>0$ as can be seen from (4.9) and (2.16). Since $\rho_{\lambda}^{(\alpha, \beta)}(x) / \Gamma(\lambda+\alpha+1)$ is an entire function in $\lambda$-in fact, it is of exponential type $\pi$-it follows from $(2.13)$ that $F^{(\alpha, \beta)}(\lambda) / \Gamma(\lambda+\alpha+1)$ is also of exponential type $\pi$. By Theorem 33 of Chapter 1 in [4], $g(t)$ is analytic in $|t|>\pi$ and

$$
\frac{F^{(\alpha, \beta)}(\lambda)}{\Gamma(\lambda+\alpha+1)}=\frac{1}{2 \pi i} \int_{\gamma} e^{\lambda t} g(t) d t
$$

where $\gamma$ is any contour containing the disc $|t| \leq \pi$.

By combining (3.2) and (4.10), we obtain

$$
\hat{f}^{(\alpha, \beta)}(z)=\frac{1}{2 \pi i} \int_{\gamma} L(t, z) g(t) d t, \quad z \notin \operatorname{supp} f,
$$

for $|\zeta|$ sufficiently small so that $\operatorname{Re} t>\ln |\zeta|$ for all $t \in \gamma$, where $z$ and $\zeta$ are related as in (2.11).

Going in the other direction, we combine (4.9), (2.13), and (2.18) to obtain

$$
\begin{aligned}
g(t) & =\frac{1}{2} \int_{0}^{\infty} e^{-t \lambda}\left\langle f(x), \rho_{\lambda}^{(\alpha, \beta)}(x)\right\rangle d \Lambda=\frac{1}{2}\langle f(x), J(t, x)\rangle \\
& =\frac{1}{2} \lim _{\varepsilon \rightarrow 0^{+}} \int_{-1}^{1}[\hat{f}(x+i \varepsilon)-\hat{f}(x-i \varepsilon)] J(t, x) d x .
\end{aligned}
$$

Now we apply Hadamard's argument to continue both (4.11) and (4.12) analytically beyond their initial domains of definition. For example, if we deform the contour $\gamma$ in (4.11) in such a way that no singularity of the integrand crosses over $\gamma$, the integral remains unchanged. Keeping this in mind, we now let $z$ move in the $z$-plane and deform the contour, if necessary, to avoid having any singularity of the integrand cross over the contour. This process can be continued until we have a singularity of the integrand threatening to cross over the contour $\gamma$ and it is no longer possible to deform $\gamma$ to avoid it. This happens whenever $\gamma$ becomes trapped between a singularity of $g(t)$ and a singularity of $L(t, z)$, i.e., whenever $g(t)$ and $L(t, z)$ have a common singular point. Since, by Corollary 4.1 , the only possible singularities of $L(t, z)$ are at $z= \pm 1$ and $z=\frac{1}{2}\left(e^{t}+e^{-t}\right)$, it follows that if $g(t)$ has a singularity at $t=t_{0}$ or $t=\bar{t}_{0}$, then $\hat{f}^{(\alpha, \beta)}(z)$ may have one at $z= \pm 1$ and $z_{0}=\frac{1}{2}\left(e^{t_{0}}+e^{-t_{0}}\right)$. With a slight modification [7, p. 1411], we can apply the same argument to (4.12) to conclude that if $\hat{f}(z)$ has a singularity at $z=z_{0}$, then $g(t)$ may have one at $t=t_{0}$ or $t=\bar{t}_{0}$ where $z_{0}=\frac{1}{2}\left(e^{t_{0}}+e^{-t_{0}}\right)$. The fact that $\hat{f}(z), \hat{f}^{(\alpha, \beta)}(z)$ have the same singularities and that these singularities are in $(-1,1)$ completes the proof. Q.E.D. 


\section{REFERENCES}

1. H. Bremermann, Distributions, complex variables and Fourier transforms, Addison-Wesley, New York. 1965.

2. E. Deeba and E. Koh, The continuous Jacobi transform, Internat. J. Math. Math. Sci. 6(1983), 145-160.

3. R. Gilbert, Integral operator methods in bi-axially symmetric potential theory, Contrib. Differential Equations 1 (1963), 441-456.

4. J. Levin, Distribution of zeros of entire functions, Amer. Math. Soc. Transl. 5 (1972).

5. Z. Nehari, On the singularities of Legendre expansions, J. Rational Mech. Anal. 5 (1956), 987-991.

6. G. Szegö, Orthogonal polynomials, 4th ed., Amer. Math. Soc. Colloq. Publ., Vol. 23, Amer. Math. Soc., Providence, R.I., 1978.

7. G. Walter, On real singularities of Legendre expansions, Proc. Amer. Math. Soc. 19 (1968), 1407-1412.

8. G. Walter and A. Zayed, On the singularities of continuous Legendre transforms, Proc. Amer. Math. Soc. 97 (1986), 673-681.

9. A. Zayed, A generalized inversion formula for the continuous Jacobi transform when $\alpha+\beta=0$ (submitted), Internat. J. Math. Math. Sci. (to appear).

10. _ A generalized inversion formula for the continuous Jacobi transform, C. R. Math., Math. Reports Acad. Sci. Canada, Vol. 8, No. 4, 1986, pp. 271-276.

Department of Mathematics, California Polytechnic State University, SAN Luis OBISPo, CALIFORNIA 93407 